\title{
Chronisches Fatiguesyndrom: Was vertreibt die anhaltende Müdigkeit?
}

\section{Das chronische Müdigkeitssyndrom ist eine Erkrankung ungeklärter Ätio- logie. Es wird eine Vielzahl von thera- peutischen Ansätzen empfohlen.}

— In einer randomisierten Parallelgruppenstudie wurden 641 Patienten mit einem chronischen Müdigkeitssyndrom in vier Gruppen randomisiert. Die erste Gruppe erhielt eine standardisierte Behandlung durch Spezialisten, die zweite Gruppe dieselbe Behandlung in Kombination mit der adaptiven Pacing-Behandlung, die dritte Gruppe eine kognitiv-behaviorale Therapie und die vierte Gruppe eine graduierte Sporttherapie. Unter adaptiver Pacing-Behandlung wird ein Behandlungskonzept verstanden, in dem in kontrollierter Weise Phasen der Belastung mit Phasen der Ruhe abwechseln.

Die Behandlung erfolgte über einen Zeitraum von 52 Wochen. Der primäre Endpunkt war die Müdigkeit, gemessen mit der Chalder-Müdigkeitsskala, und die körperliche Leistungsfähigkeit, gemessen mit der Short Form 36.

Verglichen mit der Behandlung des Spezialisten allein ergab sich sowohl für

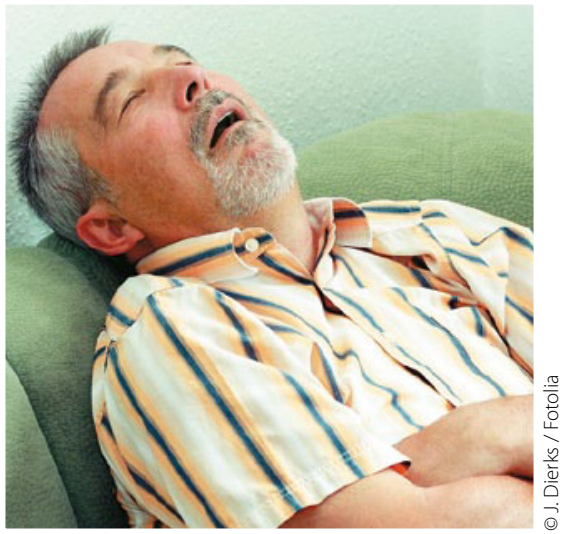

Wie bekommt man ihn wieder wach?

die kognitiv-behaviorale Therapie als auch für die graduierte körperliche Belastungsbehandlung eine signifikante Verbesserung von Müdigkeit und eine Besserung der körperlichen Leistungsfähigkeit. Der zusätzliche Einsatz der adaptiven Pacing-Behandlung war nicht besser als eine Behandlung des Spezialisten allein.

Bei der Behandlung des chronischen Müdigkeitssyndroms sind kognitiv-behaviorale Ansätze und eine graduierte körperliche Belastungstherapie als Kom- binationsbehandlung mit einer Betreuung durch Spezialisten besser wirksam als die ausschließliche Behandlung durch Spezialisten.

\section{Kommentar}

Das chronische Müdigkeitssyndrom ist einer der großen Mythen in der Medizin. Es ist nach wie vor unklar, wie dieses Krankheitsbild zustande kommt und ob es mehr ist als eine somatisierte Depression. Dessen ungeachtet gibt es aber gute wissenschaftliche Evidenz, dass eine multimodale Therapie, die eine kognitiv-behaviorale Therapie mit einem körperlichen Training verbindet, wirksam ist. Dies konnte auch in dieser großen, gut geplanten und durchgeführten randomisierten Studie belegt werden. Wichtig für die Beratung dieser Patienten ist, dass die sogenannte adaptive Pacing-Therapie keinen positiven Effekt hat.

H.-C. DIENER .

- P. D. White et al.

Comparison of adaptive pacing therapy, cognitive behaviour therapy, graded exercise therapy, and specialist medical care for chronic fatigue syndrome (PACE): a randomised trial. Lancet 377 (2011) 823-836 